\title{
Gauge invariant field strength correlators and Abelian dominance in QCD
}

\author{
Nora Brambilla ${ }^{\mathrm{a} *}$ and Antonio Vairo ${ }^{\mathrm{b}}$ \\ ${ }^{a}$ Institut für Theoretische Physik, Univ. Wien, Boltzmanngasse 5, A-1090 Vienna, Austria

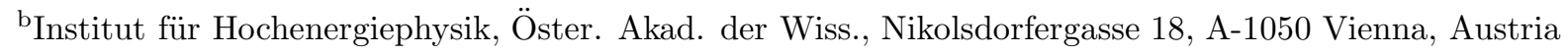

We establish a relation between the two-point field strength correlator in QCD and the dual field propagator of the effective dual Abelian Higgs model describing the infrared behaviour of QCD.

\section{INFRARED ABELIAN DOMINANCE}

The QCD infrared dynamics appears to be well approximated by a (dual) Abelian Higgs model [1.2]. Via Abelian projection the QCD gluodynamics is reduced to Abelian fields, Abelian monopoles and charged matter fields degrees of freedom. Then, lattice simulations (mainly in $S U(2)$ and in Maximal Abelian projection) show Abelian dominance and monopole dominance in the long range features of QCD. This means that nonperturbative dynamical quantities like the string tension $\sigma$ are reproduced (for more than 90\%) simply by considering the Abelian or the monopole degrees of freedom. Moreover, monopoles are condensed in the confined phase. Electric fields and magnetic currents in the presence of static sources are consistent with the dual Maxwell and Ginzburg-Landau equations. The penetration length $\lambda=1 / M(M=$ dual gluon mass) and the correlation length $\xi=1 / M_{\phi}$ $\left(M_{\phi}=\right.$ Higgs Mass $)$ are measured. Finally, the effective monopole Lagrangian is extracted from an inverse Montecarlo method and turns out to be equivalent to the Lagrangian of the dual Abelian Higgs model. Therefore, the above lattice investigations show that 1) QCD lattice gluodynamics is equivalent to the dual Abelian Higgs model: the Higgs particles are Abelian monopoles and condense in the confinement phase. 2) $M \simeq M_{\phi}$ and therefore the $\mathrm{QCD}$ vacuum behaves as a dual superconductor on the border between type I and type II.

\begin{tabular}{lllll}
\hline${ }^{*}$ Marie Curie fellow, & TMR contract & n. \\
ERBFMBICT961714
\end{tabular}

These are the information coming from the lattice. A still open problem is how to relate the Higgs and the dual gluon mass with the QCD parameters. In the following we show that an "Abelian effective dominance" shows up in the cumulant expansion of the quasi-static Wilson loop average in the nonperturbative region. This allows us to relate the non-local gluon condensate (i.e. the two-point field strength correlator) with the dual field propagator of an underlying dual Abelian Higgs model and therefore to suggest a connection between QCD and dual parameters.

\section{WILSON LOOP AND FIELD STRENGTH CORRELATORS}

In this section we report evidence of the Gaussian dominance in the cumulant expansion of the quasi-static Wilson loop average. The infrared gluodynamics is contained in the Wilson loop which is an order parameter of confinement $W(\Gamma) \equiv \mathrm{P} \exp \left\{i g \oint_{\Gamma} d z_{\mu} A_{\mu}(z)\right\}$ and $\Gamma$ is a loop made up by the two quark trajectories plus the endpoint Schwinger strings. In Euclidean space, using the non-Abelian Stokes theorem, the Wilson loop average on the Yang-Mills action, \langle\rangle$\equiv$ $\int \mathcal{D} A \exp \left\{i S_{\mathrm{YM}}[A]\right\}$, can be expanded as [3],

$\langle W(\Gamma)\rangle=\exp \sum_{n=0}^{\infty} \frac{(i g)^{n}}{n !} \int_{S(\Gamma)} d S(1) \cdots d S(n)$
$\times\langle\phi(0,1) F(1) \phi(1,0) \cdots F(n) \phi(n, 0)\rangle_{\text {cum }}$.

where $F(n) \equiv F_{\mu_{n} \nu_{n}}\left(u_{n}\right)$ is the field strength. $S(\Gamma)$ denotes a surface with contour $\Gamma$ and 
$d S(n) \equiv d S_{\mu_{n} \nu_{n}}\left(u_{n}\right)$. The cumulants \langle\rangle$_{\text {cum }}$ are defined as the connected part of the average and $\langle\phi(0,1) F(1) \phi(1,0)\rangle_{\text {cum }}=\langle\phi(0,1) F(1) \phi(1,0)\rangle=$ 0. $\phi(0, u)=\exp \left\{i g \int_{0}^{u} d z_{\mu} A_{\mu}(z)\right\}$ is a Schwinger line.

Let us consider $\left\langle\left\langle g^{2} F_{\mu \nu}(x) F_{\lambda \rho}(0)\right\rangle_{\Gamma} \quad-\right.$ $\left\langle\left\langle g F_{\mu \nu}(x)\right\rangle_{\Gamma}\left\langle\left\langle g F_{\lambda \rho}(0)\right\rangle_{\Gamma}\right.\right.$, with $\Gamma$ a rectangular Wilson loop and the points $x \equiv(\mathbf{r}, t)$ and 0 belonging to the first and second temporal quark line respectively. This object is required to compute the $1 / \mathrm{m}^{2}$ corrections to the static quarkantiquark potential and has been evaluated on the lattice. The double bracket $\left\langle\langle\rangle_{\Gamma}\right.$ stands for the average over gauge fields in presence of the Wilson loop $W(\Gamma)\left\langle\langle\rangle_{\Gamma} \equiv\langle W(\Gamma)\rangle /\langle W(\Gamma)\rangle\right.$. Taking into account Eq. (1), we obtain,

$\left\langle\left\langle g^{2} F_{\mu \nu}(x) F_{\lambda \rho}(0)\right\rangle_{\Gamma}-\left\langle\left\langle g F_{\mu \nu}(x)\right\rangle\right\rangle_{\Gamma}\left\langle\left\langle g F_{\lambda \rho}(0)\right\rangle_{\Gamma}=\right.\right.$ $g^{2}\left\langle\phi(0, x) F_{\mu \nu}(x) \phi(x, 0) F_{\lambda \rho}(0)\right\rangle+\mathcal{R}_{\mu \nu \lambda \rho}(x ; \Gamma)$,

where $\mathcal{R}$ contains all contributions coming from cumulants of order higher than two:

$$
\begin{aligned}
& \mathcal{R}_{\mu \nu \lambda \rho}(x ; \Gamma)= \\
& \sum_{n=3}^{\infty} \frac{(i g)^{n}}{n !} \sum_{\{\text {all perm. }(i, j)\}} \int_{S(\Gamma)}\left[\prod_{k \neq i, j}^{n} d S_{\mu_{k} \nu_{k}}\left(u_{k}\right)\right] \\
& \times\left\langle\phi\left(0, u_{1}\right) F_{\mu_{1} \nu_{1}}\left(u_{1}\right) \cdots F_{\mu \nu}\left(u_{i}=x\right)\right. \\
& \left.\times \cdots F_{\lambda \rho}\left(u_{j}=0\right) \cdots F_{\mu_{n} \nu_{n}}\left(u_{n}\right) \phi\left(u_{n}, 0\right)\right\rangle_{\text {cum }} .
\end{aligned}
$$

In the so-called Stochastic Vacuum Model [3] it is assumed that for large distances the bilocal cumulant $\left\langle\phi(0, x) F_{\mu \nu}(x) \phi(x, 0) F_{\lambda \rho}(0)\right\rangle$ is the dominant contribution to the r.h.s. in Eq. (2). This assumption has been (phenomenologically) successfully tested in potential theory and in soft high energy scattering processes. Eq. (2) is suitable to obtain a first principles check of this assumption on the lattice. The adopted strategy is the following [4]. Within Eq. (2) Lorentz invariance is only violated by the contour $\Gamma$. Therefore, deviations of the l.h.s. of Eq. (2) from Lorentz invariance have to be attributed to the higher order cumulants $\mathcal{R}$ on the r.h.s. We interpret the Lorentz invariant part of the data as $\left\langle\phi(0, x) F_{\mu \nu}(x) \phi(x, 0) F_{\lambda \rho}(0)\right\rangle$ and parameterize it in terms of two form factors $D$ and $D_{1}$

$$
\left\langle g^{2} \phi(0, x) F_{\mu \nu}(x) \phi(x, 0) F_{\lambda \rho}(0)\right\rangle \equiv
$$

$$
\begin{aligned}
& \left(\delta_{\mu \lambda} \delta_{\nu \rho}-\delta_{\mu \rho} \delta_{\nu \lambda}\right) D\left(x^{2}\right)+\frac{1}{2}\left[\frac{\partial}{\partial x_{\mu}}\left(x_{\lambda} \delta_{\nu \rho}-x_{\rho} \delta_{\nu \lambda}\right)\right. \\
& \left.+\frac{\partial}{\partial x_{\nu}}\left(x_{\rho} \delta_{\mu \lambda}-x_{\lambda} \delta_{\mu \rho}\right)\right] D_{1}\left(x^{2}\right) .
\end{aligned}
$$

All the dynamics is contained in the form factors. The function $D$ is responsible for the area law and confinement. Both functions $D$ and $D_{1}$ are proportional to the gluon condensate $G_{2} \equiv$ $\left\langle\alpha_{\mathrm{s}} F^{2}(0)\right\rangle / \pi$.

In Ref. [I] we have examined a set of lattice data for the 1.h.s. of Eq.(2). We have found that: 1 ) it exists a window (roughly $x$ bigger than $0.2 \div 0.3 \mathrm{fm}$ ) in which Lorentz invariance is restored in the continuum limit and therefore the r.h.s. of Eq. (2) is well approximated by the two-point field strength correlator; 2) in this window the functions $D$ and $D_{1}$ display an exponential decreasing behaviour $\simeq G_{2} \exp \left\{-|x| / T_{g}\right\}$ with a correlation length $T_{g} \simeq 0.12 \div 0.19 \mathrm{fm}$. For the details we refer to Ref. [⿴囗十. These results compare well with other lattice determinations of these quantities which have been obtained by use of the so-called cooling method 5 . However this is the first direct measurement without cooling and the first lattice evidence in favor of the Gaussian dominance in the Wilson loop average,

$\langle W(\Gamma)\rangle \simeq e^{-\frac{1}{2} \int_{S(\Gamma)} d S_{\mu \nu} \int_{S(\Gamma)} d S_{\rho \sigma}\left\langle g^{2} \phi F_{\mu \nu} \phi F_{\lambda \rho}\right\rangle}$

In the long distance region (bigger than $0.2 \mathrm{fm}$ ) the Wilson loop average is well approximated by the two-point field strength correlator and therefore the QCD infrared dynamics is "effectively Abelian". Moreover, the nonperturbative regime is controlled by two parameters: the gluon condensate $\left\langle F^{2}(0)\right\rangle$ and the gluon correlation length $T_{g}$. The static quark-antiquark potential is then given by [3]

$V_{0}(R)=\frac{1}{2} \int_{\left|x_{1}\right|<R} d^{2} x\left(R-\left|x_{1}\right|\right) D\left(x^{2}\right)+\frac{\left|x_{1}\right|}{2} D_{1}\left(x^{2}\right)$

with $d^{2} x=d x_{1} d x_{4}, x^{2}=x_{1}^{2}+x_{4}^{2}$. In the limit $r / T_{g} \gg 1, V_{0}(r) \rightarrow \sigma r$ with the string tension given by

$\sigma=\frac{1}{2} \int d^{2} x D\left(x^{2}\right)$. 
A non-vanishing $D$ function leads to confinement.

Quite naturally now a question arises. What is the relation between the "Abelian behaviour" of the Wilson loop average discussed above and the effective dual Abelian Higgs model suggested by the infrared lattice data mentioned in Sec. 1?

\section{DUAL EFFECTIVE DYNAMICS}

In Sec. 1 we have reported that the infrared dynamics of QCD seems to be effectively described by a dual Abelian Higgs model with action

$$
\begin{aligned}
& S\left(C_{\mu}, \phi\right)=\int d^{4} x\left[\frac{1}{4} G_{\mu \nu}(x) G_{\mu \nu}(x)\right. \\
& \left.+\frac{1}{2}\left(D_{\mu} \phi\right)^{*}(x)\left(D_{\mu} \phi\right)(x)+V\left(\phi^{*}(x) \phi(x)\right)\right]
\end{aligned}
$$

where $G_{\mu \nu}(x)=\partial_{\mu} C_{\nu}(x)-\partial_{\nu} C_{\mu}(x)+$ $G_{\mu \nu}^{s}(x), C_{\mu}$ being the dual field and $G_{\mu \nu}^{s}=$ $g \varepsilon_{\mu \nu \alpha \beta} \int_{0}^{1} d \tau \int_{0}^{1} d \sigma \frac{\partial y_{\alpha}}{\partial \sigma} \frac{\partial y_{\beta}}{\partial \tau} \delta^{4}(x-y(\tau, \sigma))$ the Dirac field strength containing the quark currents flowing around the Wilson loop. $\phi$ is the Higgs field and $V\left(\phi^{*} \phi\right)=\frac{\lambda}{4}\left(\phi^{*} \phi-\phi_{0}^{2}\right)^{2}$ is the Higgs potential. The Higgs field is coupled to the gauge field $C_{\mu}$ via the covariant derivative $D_{\mu} \phi=\left(\partial_{\mu}+i e C_{\mu}\right) \phi$. The parameters of the model are the dual coupling constant $e=2 \pi / g$, the Higgs coupling constant $\lambda$ and the Higgs vacuum expectation value $\phi_{0}$. The classical equations of motion follow from the action (6). We emphasize that in this dual description the classical approximation does apply.

We have now two effective Abelian descriptions of the Wilson loop average: Eqs. (3)(4) and Eq. (6). These are discussed in Ref. [6]. There the two-point field strength correlator (3) is related to the corresponding dual quantity $\stackrel{\mathcal{G}}{\sigma \gamma \lambda \rho}(x, y) \equiv\left(\delta_{\lambda \sigma} \delta_{\rho \gamma}-\delta_{\lambda \gamma} \delta_{\rho \sigma}\right) \delta^{4}(x-y)-$ $\epsilon_{\mu \nu \lambda \rho} \epsilon_{\beta \alpha \sigma \gamma} \partial_{\beta}^{y} \partial_{\mu}^{x} \mathcal{K}_{\nu \alpha}(x, y)$, where $\mathcal{K}_{\mu \nu}$ is the dual field propagator, and therefore to the underlying dual superconductor mechanism. We summarize here the main results.

\subsection{London limit without quark sources}

Let us consider the pure vacuum without quark sources. In the case $\lambda \rightarrow \infty$ we have $\phi=\phi_{0}$ and the Higgs field part of the action decouples. The dual field propagator $\mathcal{K}_{\mu \nu} \equiv\left\langle C_{\mu} C_{\nu}\right\rangle$, satisfies the equation:

$\left(\partial^{2} \delta_{\nu \mu}-\partial_{\nu} \partial_{\mu}-e^{2} \phi_{0}^{2} \delta_{\nu \mu}\right) \mathcal{K}_{\nu \alpha}(x, y)=-\delta_{\mu \alpha} \delta^{4}(x-y)$

with solution ( $\infty$ stands for London limit)

$\begin{aligned} \mathcal{K}_{\mu \alpha}^{\infty}(x, y) & =\left(\delta_{\mu \alpha}-\frac{\partial_{\mu} \partial_{\alpha}}{M^{2}}\right) \mathcal{K}^{\infty} \\ \mathcal{K}^{\infty}(x, y) & =\frac{M}{(2 \pi)^{2}} \frac{K_{1}(M x)}{x}\end{aligned}$

with $M=e \phi_{0}$ the mass of the dual gluon. As a consequence we obtain

$\mathcal{G}_{\sigma \gamma \lambda \rho}(x)=\left(\delta_{\lambda \sigma} \delta_{\rho \gamma}-\delta_{\lambda \gamma} \delta_{\rho \sigma}\right) D^{\infty}\left(x^{2}\right)+\frac{1}{2}\left[\frac{\partial}{\partial x_{\lambda}}\right.$

$\left.\left(x_{\sigma} \delta_{\rho \gamma}-x_{\gamma} \delta_{\rho \sigma}\right)+\frac{\partial}{\partial x_{\rho}}\left(x_{\gamma} \delta_{\lambda \sigma}-x_{\sigma} \delta_{\lambda \gamma}\right)\right] D_{1}^{\infty}\left(x^{2}\right)$

with $D^{\infty}\left(x^{2}\right)=\frac{M^{3}}{4 \pi^{2}} \frac{K_{1}(M x)}{x}, \quad D_{1}^{\infty}\left(x^{2}\right)=$ $-4 \frac{\mathrm{d}}{\mathrm{d} x^{2}} K^{\infty}\left(x^{2}\right)$. Therefore, assuming the London limit of a dual Abelian Higgs action as a model of infrared QCD, the predicted form of the twopoint field strength correlator as in Eq. (3), manifests a non-vanishing function $D$ and an exponential fall off for the functions $D, D_{1}$. Since $\mathcal{K}^{\infty}\left(x^{2}\right) \underset{|x| \rightarrow \infty}{\longrightarrow} \frac{1}{2} \frac{1}{(2 \pi)^{3 / 2}} \frac{1}{\sqrt{M} x^{3 / 2}} e^{-M x}+\cdots$, the dual gluon mass $M$ plays the role of a correlation length.

The logarithmic divergence of the string tension calculated via (5) shows that the London limit is unphysical near quark sources where the Higgs field cannot be considered freezed to his vacuum value. In a physical situation we have to consider quarks and a Higgs field which vanishes on the Dirac string.

\subsection{Dual dynamics with quark sources}

Let us identify in the long range

$$
W(\Gamma) \simeq\left\langle e^{-S\left(C_{\mu}, \phi\right)}\right\rangle,
$$

with the action $S$ given by Eq. (6) and containing quark sources via the Dirac string. Using the classical equations of motion we find

$$
S\left(C_{\mu}^{\mathrm{cl}}, \phi^{\mathrm{cl}}\right)=\frac{1}{2} \int_{S(\Gamma)} d S_{\sigma \gamma}(v) \int_{S(\Gamma)} d S_{\lambda \rho}(u)
$$




$$
\times \mathcal{G}_{\sigma \gamma \lambda \rho}(v, u)+\int d^{4} x\left[\frac{1}{2}(\partial \phi(x))^{2}+V\left(\phi^{2}(x)\right)\right] .
$$

However, neglecting the dependence of the Higgs field, via the equations of motion, on the strings, taking into account the contribution coming from the Higgs part as a finite contribution to the string tension, we find that, also in the general case, $\mathcal{G}_{\sigma \gamma \lambda \rho}$ is equivalent to the QCD two-point non-local condensate (3) and in principle gives information on the validity of the decomposition (3) and on the existence and the behaviour of the functions $D$ and $D_{1}$. In the approximation in which the Higgs field depends only on the transverse coordinates, $\mathcal{G}$ depends only on the parallel components. In this approximation we can evaluate analytically the electric component of $\mathcal{G}$ and find that its behaviour is controlled by a form factor $D(x)=\frac{1}{2 \pi \ell^{2}} K_{0}\left(\frac{|x|}{\ell}\right) \underset{x \rightarrow \infty}{\rightarrow} \sqrt{\frac{\pi}{2} \frac{\ell}{|x|}} e^{-|x| / \ell}$ where $\ell=\sqrt{S_{c}} / S_{\phi}$, and $S_{c}=\lim _{x_{\perp} \rightarrow 0} e C^{\mathrm{np}}\left(x_{\perp}\right) / x_{\perp}$ and $S_{\phi}=\lim _{x_{\perp} \rightarrow 0} e \phi\left(x_{\perp}\right) / x_{\perp}$ are constants known in terms of the Ginzburg-Landau parameter $\lambda / e^{2}$. This suggests the identification of the correlation length $T_{g}$ with the dual quantity $\ell$. Notice that at variance with respect to the London limit result, here the correlation length is not simply given by the mass $M$ of the dual gluon.

The static potential can be calculated exactly without the use of an ultraviolet cut-off

$$
\begin{aligned}
& V_{0}(R)=-\frac{g^{2}}{4 \pi} \frac{1}{R}+\frac{g^{2}}{2 \pi} S_{c} \int_{0}^{R} d x_{1} 2\left(R-x_{1}\right) \\
& \times \int_{-\infty}^{+\infty} d x_{4} \frac{1}{2 \pi \ell^{2}} K_{0}\left(\frac{\sqrt{x_{4}^{2}+x_{1}^{2}}}{\ell}\right)+\text { Higgs contr. } \\
& =R \frac{g^{2}}{2 \pi} S_{c}+\left(e^{-R / \ell}-1\right) \frac{g^{2}}{2 \pi} S_{c} \ell-\frac{g^{2}}{4 \pi} \frac{1}{R}+R \sigma_{\mathrm{H}} \\
& \underset{R \rightarrow \infty}{\longrightarrow} R \frac{g^{2}}{2 \pi} S_{c}+R \sigma_{\mathrm{H}},
\end{aligned}
$$

being the Higgs contribution to the static potential given by a linear term with string tension $\sigma_{\mathrm{H}}$. Taking explicitly into account this contribution, the total string tension is $\sigma=$ $\frac{g^{2}}{2 \pi} S_{c}+\sigma_{\mathrm{H}}$. In particular, for a superconductor on the border $\left(\lambda / e^{2}=1 / 2\right)$ we have $V_{0}(R)=$ $\pi \phi_{0}^{2}\left(R+\ell\left(e^{-R / \ell}-1\right)\right)-\frac{g^{2}}{4 \pi} \frac{1}{R}$. It is also possible to relate the Higgs condensate to the gluon condensate $G_{2} \simeq \lambda \phi_{0}^{4}$.

\section{CONCLUSIONS}

In the assumption that the infrared behaviour of QCD is described by an effective Abelian Higgs model we have related the nonperturbative behaviour of the two-point field strength correlator $\left\langle g^{2} F_{\mu \nu}(x) \phi(x, y) F_{\lambda \rho}(y) \phi(y, x)\right\rangle$ in QCD with the dual field propagator in the effective Abelian Higgs model of infrared QCD. In this way the origin of the non-local gluon condensate is traced back to an underlying Meissner effect and the phenomenological relevance of the Gaussian approximation on the Wilson loop is understood as following from the classical approximation in the dual theory of long distance QCD. In particular the correlation length $T_{g}$ of $\mathrm{QCD}$, which we know from direct lattice measurements, can be expressed completely in terms of the dual theory parameters. We have calculated analytically the static potential and the string tension. It turns out that the string tension is given by an integral over a function of the correlation length which can be identified with the non-local gluon condensate. There is no cut-off introduced in this calculation since it is not performed in the London limit.

\section{REFERENCES}

1. M. Polikarpov in Proceedings of "Quark Confinement and the Hadron Spectrum III", World Scientific, Singapore, 1998, and Refs. therein.

2. G. S. Bali in Proceedings of "Quark Confinement and the Hadron Spectrum III", World Scientific, Singapore, 1998, and Refs. therein.

3. H. G. Dosch, Phys. Lett. B 190 (1987) 177; H. G. Dosch and Yu. A. Simonov, Phys. Lett. B 205 (1988) 339.

4. G. S. Bali, N. Brambilla and A. Vairo, Phys. Lett. B 421 (1998) 265.

5. M. D'Elia, A. Di Giacomo, and E. Meggiolaro, Phys. Lett. B 408 (1997) 315.

6. M. Baker, N. Brambilla, H. G. Dosch and A. Vairo, Phys. Rev. D 58 (1998). 